\title{
Audit légal et perception de la qualité des travaux dans une économie en développement
}

\author{
Serge Valant GANDJA
}

Professeur, BEM-Bordeaux Ecole de Management

Résumé : S'appuyant sur les théories de l'agence, d'asymétrie informationnelle et des parties prenantes (stakeholder theory), cet article développe et teste un modèle explicatif de la perception de la qualité des missions d'audit légal chez les utilisateurs des états financiers. Les données collectées auprès des commissaires aux comptes, inspecteurs des impôts, analystes de crédits, directeurs financiers des villes de Douala et

\begin{abstract}
This study highlights the perceived quality of audit work by the legal users of financial statements. It combines the contributions of agency and stakeholder theories. By a positive approach, final samples of 109 questionnaires were collected from auditors, tax inspectors, credit analysts, financial managers of the cities of Douala and Yaounde. Results from statistical processing
\end{abstract}

Yaoundé, ont subi des traitements à travers un modèle logit binaire. Il ressort que la situation financière de l'audité, la capacité à révéler les anomalies, la réputation du cabinet et la gestion attendue de la mission apparaissent comme des vecteurs majeurs de cette perception.

Mots clés : qualité perçue - audit légal indépendance - compétence - états financiers

emphasize that listeners must have a qualification and sufficient experience to carry out their mission. Also, special attention should be given to the auditee's financial position, reputation and firm size, and level of control over the profession.

Key words: perceived quality - statutory audit independence - competence - financial statements 


\section{Introduction}

La série de scandales financiers ${ }^{1}$ qui a eu lieu à l'aube du $21^{\mathrm{e}}$ siècle, amplifiée par la survenance d'autres « affaires » d'envergure tels que Worldcom, Parmalat, Vivendi et, plus récemment, le cas Maddof, ajoutée aux manipulations non révélées du trader de la Société générale des banques, justifient la crise de confiance constatée des utilisateurs de l'information comptable et financière et discréditent les cabinets d'audit garants de son intégrité.

Face à ce malaise grandissant, des mesures ont été prises visant à réorganiser et à renforcer les règles de gouvernance d'entreprise, d'une part, le système de contrôle de la qualité de l'audit, d'autre part. C'est le cas notamment avec la loi Sarbannes-Oxley aux Etats-unis, la loi de Sécurité financière en France ou la $8^{\mathrm{e}}$ Directive dans l'Union européenne. Toutefois, le Cameroun n'est pas resté en marge de cette mouvance. En atteste la loi n²003/008 du 10 juillet 2003 relative à la répression des infractions contenues dans certains actes uniformes OHADA. Afin de décourager les comportements déliquescents, celle-ci prévoit dans son article 16 des sanctions allant de 2 à 5 ans d'emprisonnement et/ou une amende de 200 mille à 5 millions de francs CFA, pour sanctionner tout acte de nature à porter atteinte à l'indépendance et aux incompatibilités des auditeurs externes.

En dépit des mesures prises sur la scène nationale camerounaise, facilitées par l'« Opération Epervier $»^{2}$, on constate au sein des entreprises étatiques l'inculpation de membres de l'Ordre national des experts comptables camerounais (ONECCA) pour complicité de détournement des deniers publics ${ }^{3}$. Ces interpellations contribuent à discréditer plus encore la profession comptable libérale au Cameroun.

Il est donc vital de crédibiliser en permanence la qualité des travaux d'audit afin de rassurer les utilisateurs de l'information comptable et financière désillusionnés par les scandales financiers devenus quasi permanents. L'institution de règles de gouvernance crédibles ne pouvant se faire que sur la base d'une prise en compte des attentes des parties prenantes de

\footnotetext{
${ }^{1}$ Marquée par l'affaire Enron et la faillite de Global Crossing dont Andersen LLP était l'auditeur.

${ }^{2}$ L'Opération Epervier est le nom donné par le gouvernement camerounais à l'entreprise d'assainissement des pratiques de gestion dans les sociétés d'Etat et les administrations publiques camerounaises, lancée en février 2006 (Nouvelle expression, 2007).

${ }^{3}$ Sept membres de l'Ordre national des experts comptables camerounais (ONECCA) sont arrêtés et emprisonnés ; deux commissaires aux comptes sont condamnés pour détournement de deniers publics sur la base des articles 74 et 184 du Code pénal.
} 
l'activité d'audit, une analyse empirique de la qualité perçue des travaux d'audit s'avère opportune au Cameroun. Si grâce à l'auditeur on peut se fier à la qualité des comptes présentés par une entreprise, une question demeure : comment peut-on savoir si l'on peut faire confiance à l'auditeur lui-même ? Le doute persiste surtout lorsqu'on on a connaissance de l'autonomie et de la marge de manœuvre technique laissées aux firmes d'audit au Cameroun ${ }^{4}$.

Cette étude se propose d'identifier dans le contexte camerounais les facteurs susceptibles d'influencer la perception de la qualité des missions de l'auditeur. Elle mobilise dans une première partie une littérature sur les théories de l'agence, d'asymétrie informationnelle et, enfin, des parties prenantes. Tout d'abord, les contributions de l'audit à la résolution des problèmes d'agence et d'asymétrie informationnelle sont mises en évidence. En effet, partant de l'hypothèse d'information imparfaite, la théorie de l'agence relève une incomplétude des contrats et montre que le principal ne dispose pas des moyens nécessaires pour contrôler l'action de l'agent (Coriat et Weinstein, 1995). Elle souligne ainsi l'existence d'une relation entre l'entreprise et les lecteurs de comptes, entre l'auditeur et les utilisateurs des états financiers. L'étude montre ensuite que la théorie des parties prenantes (ou stakeholder theory) accorde un poids considérable aux signes des résultats (bénéfice ou perte) et révèle la présence éventuelle d'une réserve dite $\mathrm{d}^{\prime}$ « incertitude » dans les annexes, souvent absente dans les rapports d'audit. Le second volet de cette première partie de l'article met en évidence les différentes relations établies dans les études empiriques antérieures et débouche sur la construction du modèle de recherche. La seconde partie positionne la méthodologie d'investigation retenue. S'inscrivant dans une approche positiviste, un échantillon final de 109 questionnaires a été collecté auprès des commissaires aux comptes et inspecteurs des impôts, des analystes de crédits et directeurs financiers des villes de Douala et de Yaoundé. L'article conclut sur la présentation et la discussion des résultats, en soulignant leurs implications managériales. Au terme des analyses en composantes principales réalisées, neuf facteurs principaux ont émergé et sont utilisés comme variables manifestes dans la régression logistique effectuée. Les résultats obtenus indiquent que les auditeurs doivent posséder des connaissances, une formation, une qualification et une expérience suffisante pour mener à bien un audit financier. Nous rejoignons dans ce sens Flint (1988), Lee (1993) et Herrbach (2000). Il ressort également que les utilisateurs des états financiers doivent accorder une

\footnotetext{
${ }^{4}$ Les initiatives d'organisation et de contrôle de la profession d'audit externe sont dévolues au seul Conseil de l'ONECCA ; aucune structure administrative n'est garante du suivi permanent du respect des incompatibilités et du suivi des dérapages ; il n'existe pas de séparation institutionnelle entre les commissaires aux comptes et les experts comptables, ni même de dispositif normatif et déontologique propre à la profession d'audit au Cameroun.
} 
attention particulière à la situation financière de l'audité, à la réputation du cabinet, au niveau de contrôle de la profession, à la rigueur du dispositif juridique et à la taille du cabinet. Ces résultats corroborent ainsi ceux de nombre d'auteurs (DeAngelo, 1981 ; Knapp, 1985 ; Watts et Zimmerman, 1986 ; Palmrose, 1987 ; Richard, 2003 ; Hazgui et al., 2010).

\section{Fondements théoriques et modèle de recherche}

Cette partie vise à positionner l'étude par rapport aux travaux antérieurs. Elle ambitionne de montrer en premier lieu l'importance de l'audit, puis, de construire le modèle théorique de recherche.

\subsection{La demande d'audit, un palliatif aux problèmes d'agence et d'asymétrie informationnelle}

Généralement exécutée dans un environnement de règles imposées par un professionnel indépendant, la mission de l'auditeur consiste à exprimer une opinion indépendante sur les états financiers établis selon des règles bien définies ${ }^{5}$. Pour l'auditeur externe, il s'agit de comprendre l'évaluation à travers l'appréciation des contrôles internes et du fonctionnement du système d'information de l'entreprise. L'audit externe devient ainsi un moyen de renforcer les mécanismes mis en œuvre par les actionnaires pour réduire les coûts d'agence et contrôler la gestion des dirigeants (Audousset-Coulier, 2008). Cependant, des situations conflictuelles d'intérêts subsistent à plusieurs niveaux dans cette relation d'agence tripolaire (entre mandants, auditeurs et dirigeants) et leur connaissance justifierait les craintes des uns et des autres.

L'objet de ce point est d'une part de mettre en évidence ces situations conflictuelles et les facteurs induits, ce à la lumière des théories appliquées à l'audit et, d'autre part, de définir leur incidence hypothétique sur la perception des parties prenantes.

\subsubsection{La résolution des problèmes d'agence via l'audit}

$\mathrm{Si}$, dans la théorie de la firme, l'entreprise est le centre d'un ensemble de contrats qui lient des intervenants aux intérêts souvent divergents, dans la théorie de la réglementation, le processus politique est vu comme une lutte entre des personnes qui souhaitent maximiser leurs utilités

\footnotetext{
${ }^{5}$ C'est le cas notamment des principes comptables généralement admis et des normes professionnelles en vigueur.
} 
personnelles par des transferts de richesse. Chaque contrat se caractérise par l'hypothèse de l'information imparfaite et par la présence d'une asymétrie d'informations entre le principal et l'agent, lequel en sait normalement davantage que le premier sur l'activité. La conséquence de ces problèmes d'information est, d'une part, que le contrat qui lie les parties est nécessairement incomplet et, d'autre part, que le principal n'a pas les moyens de contrôler parfaitement l'action de l'agent (Coriat \& Weinstein, 1995). Seuls les dirigeants ont un accès direct réel à l'activité de l'entreprise. Ceci leur confère une liberté d'action et un avantage informationnel important. Une telle situation nécessite la mise en place de moyens (incitations et contrôle $)^{6}$ destinés à orienter leur comportement, autrement dit, à assurer un minimum de convergence d'intérêts entre actionnaires et dirigeants.

\section{Les différents niveaux de conflits d'intérêts}

La littérature comptable a souvent considéré l'audit comme un élément essentiel de l'équilibre contractuel de la firme, puisqu'il permet non seulement la résolution des conflits d'agence, mais aussi de faire coïncider les intérêts des différents intervenants et de limiter les manipulations comptables. On retrouve des conflits d'intérêts dans la relation entre l'entreprise et les lecteurs de comptes et dans la relation entre l'auditeur et les utilisateurs des états financiers.

\section{- Entre l'entreprise et les lecteurs de comptes}

L'audit financier s'insère dans la relation d'agence autour de l'entreprise en tant que processus de contrôle des comptes établis par l'entreprise pour lever l'asymétrie d'informations entre les dirigeants et les autres intervenants.

Selon Jensen et Meckling (1976), la divergence d'intérêts qui peut apparaître entre dirigeants salariés (agents) et actionnaires (principal, propriétaire de l'entreprise) génère certains coûts appelés «coûts d'agence ${ }^{7}$. A titre d'exemple, le coût de surveillance représente l'ensemble des dépenses engagées par les dirigeants pour satisfaire les exigences des créanciers et notamment les banquiers. Ces derniers ont en effet l'habitude d'exiger aux sociétés qui sollicitent auprès d'eux des crédits bancaires de soumettre leurs états financiers au contrôle d'auditeurs indépendants afin de s'assurer que les dirigeants n'ont pas manipulé les chiffres comptables et qu'ils n'ont pas transféré la richesse des actionnaires à leur profit.

\footnotetext{
${ }^{6} \mathrm{Si}$ les moyens d'incitation peuvent être assimilés à la rémunération de la performance, le contrôle passe par la mise en place de procédures de suivi de ladite performance.

${ }^{7}$ Il s'agit du coût de surveillance, du coût de signalisation et du coût résiduel supportés par les actionnaires afin de faire converger les intérêts.
} 
- Entre l'auditeur et les utilisateurs des états financiers

Les cabinets d'audit étant des agents capitalistes dont l'objectif est la maximisation de leur utilité, l'activité d'audit peut elle-même être assimilée à une relation d'agence entre les utilisateurs des comptes certifiés et les cabinets (Herrbach, 2000). Dans cette relation, les utilisateurs des états financiers certifiés (principal) confient au cabinet d'audit (agent) la responsabilité de la certification des comptes de l'entreprise sans avoir accès au système d'information comptable de l'entreprise, ni au travail d'audit qui sert de support à la certification. L'audit apparaît ainsi comme un élément incontournable de la gouvernance d'entreprise, qui se doit tout au moins de paraître fiable par l'émission de signaux positifs à l'endroit des utilisateurs des états financiers.

A partir de la figure 1, on peut visualiser la place de l'audit financier dans la relation d'agence autour de l'entreprise.

Figure 1: Le rôle de l'audit financier dans la relation d'agence de l'entreprise

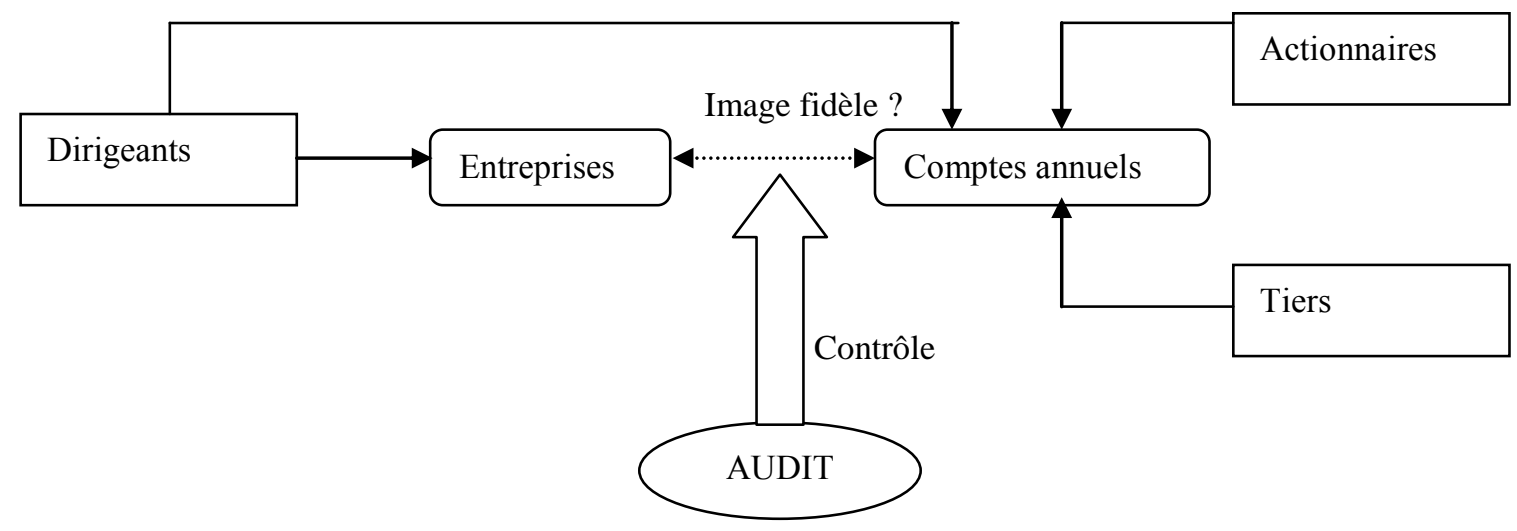

Source : Adaptée de Herrbach (2000)

En tant que garant de la bonne qualité de l'information financière, l'audit apparaît ainsi comme un élément essentiel du fonctionnement de la vie économique. Cependant, l'introduction d'un acteur supplémentaire dans une relation d'agence en tant que contrôleur ne peut résoudre complètement les problèmes liés à cette relation, surtout si cet agent est opportuniste. Comme le souligne Herrbach (2000), «le contrôleur n'est pas une entité éthérée et désintéressée, mais un acteur économique à part entière avec des finalités et des intérêts propres. En l'occurrence, l'audit est aujourd'hui le plus souvent réalisé par des gros cabinets de commissaires aux comptes ou cabinets d'audit. Ces cabinets ont un poids économique non négligeable et constituent un pôle d'influence dont les intérêts propres ne peuvent être négligés. » 
Des développements précédents, il découle trois sources de conflits : les politiques de dividende, d'endettement ${ }^{8}$ et d'investissement (Jensen et Meckling, 1976). Pour ces auteurs, les actionnaires d'une entreprise endettée peuvent être amenés à suivre une politique d'investissement sous-optimale en rejetant des projets à valeur actuelle nette positive dont les avantages reviendraient essentiellement aux agents.

\section{Comment susciter la confiance des utilisateurs de l'information comptable et financière?}

Il ne s'agit pas ici de s'attarder sur le rôle des états financiers, mais de présenter les enjeux de l'audit à partir de la mise en évidence des signaux attendus par les utilisateurs des états certifiés pour revigorer leur confiance.

Pour les actionnaires, les comptes annuels servent à déterminer la valeur de leur participation dans l'entreprise. Les dirigeants quant à eux voient de plus en plus leur rémunération déterminée, au moins pour partie, par les résultats financiers de l'entreprise qu'ils dirigent. L'Etat et les autres autorités publiques utilisent les informations comptables comme base de calcul pour la détermination des impôts et taxes qui leur sont dus. Concernant les relations d'affaires de l'entreprise (banquiers, clients, fournisseurs), elles analysent l'information financière afin de déterminer la solvabilité de leurs partenaires.

Si la nécessité de procéder à l'établissement et à la diffusion des comptes annuels dans un système économique capitaliste apparaît ainsi évidente, une telle situation pose néanmoins deux problèmes majeurs. Le premier concerne la pertinence intrinsèque des données comptables pour refléter la performance d'une entreprise. Cette question, qui a fait l'objet de multiples recherches dans le domaine de la comptabilité, ne sera pas considérée ici. Par contre, le second problème touche à la fiabilité des comptes annuels, c'est-à-dire, la mesure dans laquelle ils sont fidèles aux normes comptables de constitution et de présentation, indépendamment de la pertinence intrinsèque de ces normes. C'est ce deuxième aspect qui sera examiné dans cet article.

Il se trouve que les comptes annuels sont largement établis par les personnes même que l'on cherche à contrôler : les dirigeants de l'entreprise. La latitude dont ils disposent peut laisser planer un doute sur la sincérité de l'information qu'ils diffusent, illustré tout particulièrement par la notion de « comptabilité créative » (Gillet, 1998). L'importance de disposer de données

\footnotetext{
${ }^{8}$ Un niveau d'endettement excessif entraîne un risque de faillite. Ainsi, une mauvaise anticipation du niveau d'endettement peut être source de conflits.
} 
fiables sur les comptes annuels explique alors l'apparition de moyens pour vérifier les états financiers produits par les dirigeants à destination de l'extérieur.

Concernant les attentes des utilisateurs des états certifiés face aux motivations des dirigeants et des auditeurs, soulignons que les agents (auditeurs et dirigeants) ont intérêt à manifester leur objectivité et bonne foi dans la réalisation des tâches qui leur sont confiées par le principal. Pour Ng et Stoeckenius (1979), plusieurs conditions peuvent amener le dirigeant à choisir une fonction de reporting compatible avec les intérêts de l'actionnaire :

- $\quad$ Il doit exister un niveau d'audit capable de détecter les erreurs de reporting quelles qu'elles soient ;

- $\quad$ L'auditeur n'émet pas de réserves lorsque les rapports financiers ne le nécessitent pas ;

- $\quad$ Un meilleur niveau d'audit permet de détecter plus d'erreurs ;

- $\quad$ Le système de rémunération est basé sur les performances comptables.

Ces auteurs en concluent que la réussite de l'audit est intimement liée, non pas aux seules caractéristiques de l'audit, mais également au système de rémunération du dirigeant. $\mathrm{Ce}$ dispositif doit non seulement prendre compte des performances comptables, mais surtout prévoir une fonction de pénalité permettant de sanctionner le dirigeant qui prépare des états financiers non conformes à la réalité. Or, même en respectant les principes comptables généralement admis, on peut toujours établir des états financiers qui favorisent les objectifs des dirigeants. Comme l'ont démontré Dumontier et Raffournier (1989), la notion d'image fidèle soulève un débat entre les auditeurs, d'autant que la réglementation comptable laisse toujours des marges de manœuvre aux dirigeants pour gérer leurs résultats.

Ce cadre théorique explique l'importance de l'audit même en l'absence d'un cadre réglementaire obligeant à y recourir. Or, Ng et Stoeckenius (1979) relèvent des lacunes dans leur modèle. En effet, cette théorie explicitant la demande d'audit est incomplète parce qu'elle ignore le problème de motivation de l'auditeur afin de comprendre son comportement (Antle, 1982). L'auditeur doit être alors considéré comme un agent dont le but est la maximisation d'utilité. C'est ce qui a conduit Antle (1982), dans le cadre d'asymétrie informationnelle, à développer un modèle englobant l'actionnaire, le dirigeant et l'auditeur. Pour l'auteur, l'actionnaire ne peut guère connaître les revenus réalisés par son entreprise. Il est cependant en mesure de connaître la valeur des revenus qui apparaît dans les états financiers préparés par le dirigeant. En acceptant sa mission, l'auditeur se trouve face à un problème de reporting similaire à celui du dirigeant. Il convient alors de savoir si l'on peut accorder toute confiance 
à l'auditeur. Ce dernier peut, dans certaines conditions, être incité à élaborer un rapport non conforme à l'exigence de sincérité. Les dirigeants peuvent trouver des arguments pour l'influencer dans cette perspective. De cette situation émergent deux stratégies : celle de l'actionnaire et celle de l'auditeur.

L'actionnaire se trouve devant un problème de motivation de l'auditeur. Si l'action de l'auditeur n'est pas observable, il est nécessaire de le motiver afin qu'il rende compte de la réalité de ses conclusions. Cependant, si l'actionnaire suppose que le vérificateur qu'il a engagé est fortement indépendant, il rémunérera le dirigeant et l'auditeur sur cette base. De son côté, l'auditeur a tout intérêt à ne fournir aucun effort et à n'émettre aucune réserve (Antle, 1984). L'auditeur et le dirigeant peuvent en effet atteindre n'importe quelle incitation proposée par l'actionnaire à travers les revenus supplémentaires suite à leur collusion. Dans ce cas, ils se mettront d'accord au détriment de l'actionnaire. Face à une telle situation, l'actionnaire peut toujours craindre l'impossibilité pour l'auditeur de préserver son indépendance et décider, soit de vendre / louer son entreprise au dirigeant, soit de la liquider.

Nous avons jusqu'ici expliqué le recours par les entreprises à l'audit pour les problèmes de conflits d'intérêts et d'asymétrie d'informations. Cependant, le doute plane quant à la personnalité de l'auditeur au Cameroun. Elément essentiel du modèle proposé par Antle (1982), l'indépendance constitue le choix de l'auditeur, lequel opte pour la stratégie maximisant l'utilité de l'actionnaire. D'ailleurs, aucun indice selon l'auteur ne permet d'avancer que l'auditeur choisit une stratégie indépendante. Ceci remet en cause la qualité des travaux d'audit et explique les propos d'Archavlis et Richard (1957) en ces termes : «parmi les règles professionnelles, celle de l'indépendance est la plus importante; elle comprend et conditionne toutes les autres ». Les législateurs et les organismes professionnels sont intervenus pour protéger cette indépendance et garantir la qualité de l'audit.

\subsubsection{La théorie des parties prenantes ${ }^{9}$ et la perception de la qualité des travaux d'audit}

Sans toutefois refaire un exposé complet de cette théorie, laquelle a fait l'objet de nombre d'études, il convient ici d'en extraire la substance et d'insister surtout sur l'impact informatif du rapport d'audit sur les lecteurs de comptes.

En matière de contenu informationnel du rapport d'audit, de nombreuses voies d'investigation ont été empruntées et plusieurs réserves ont été émises. Certains chercheurs (Baskin, 1972 ;

\footnotetext{
${ }^{9}$ Tout groupe ou individu qui peut affecter ou être affecté par l'atteinte des buts de l'organisation (Freeman, 1984).
} 
Elliott, 1982 ; Dodd et al., 1984 ; Soltani, 1996) ont consacré leurs travaux à l'analyse de l'impact de la réserve liée aux changements de méthodes ou de principes comptables. Cette question a fait l'objet de débats très controversés aux Etats-Unis. Par exemple, Baskin (1972) a mené une recherche empirique sur la Bourse de New York, en recensant toutes les entreprises ayant opéré un changement de méthode comptable entre 1966 et 1969. Afin d'examiner le contenu informationnel perçu relatif aux changements de méthodes comptables sous l'hypothèse du marché efficient, l'auteur a sélectionné les firmes dont le rapport d'audit portait la mention de cette exception. Parallèlement, certaines recherches (Dodd et al., 1984) s'inscrivent dans le débat touchant au contenu informationnel de la réserve subject to, dans laquelle l'auditeur exprime l'opinion selon laquelle les états financiers sont fiables en dépit de certaines incertitudes dont l'effet n'a pas été correctement intégré lors de l'établissement des comptes de l'entreprise.

Dans une étude différente des précédentes, des auteurs ont tenté de fournir une preuve empirique de la distribution des prix de marché des actions conditionnées par l'aspect quantitatif et qualitatif des résultats comptables. Cette recherche prend en considération deux variables supplémentaires non retenues dans les autres études : le signe des résultats (bénéfice ou perte) et la présence éventuelle de la réserve dite «d'incertitude » dans l'annexe, alors que le rapport d'audit ne la contient pas.

D'autres chercheurs encore ont comparé l'influence sur les rendements boursiers de différents rapports d'audit contenant divers types de réserves. Firth (1978) fut parmi les premiers à réaliser une étude portant sur l'impact de la divulgation de l'opinion de l'auditeur avec réserves sur le prix des actions dans l'environnement britannique. Pour ce faire, l'auteur a observé les rendements des titres de toutes les sociétés britanniques cotées ayant fait l'objet d'une réserve entre 1974 et 1975. Il a ainsi analysé l'influence de quelques réserves parmi les plus couramment exprimées en Grande-Bretagne. Parmi celles-ci, on trouve : l'image fidèle, l'audit des filiales et la non-conformité avec les principes comptables.

Plus récemment, Soltani (1996) a réalisé une recherche similaire sur le marché français en observant les conséquences boursières de toutes les réserves publiées par les commissaires aux comptes des sociétés cotées à Paris entre 1980 et 1990. Dans cette étude, les opinions exprimées par les auditeurs sont réparties en quatre grands groupes : les rapports sans réserves et sans observations ; les rapports avec réserves ; les rapports avec observations, remarques et constatations ; enfin, les rapports avec « refus de certification ». 
Fields et Wilkins (1991) ont quant à eux choisi d'orienter leurs travaux dans une direction opposée à l'ensemble des études sus-citées. Au lieu de mesurer l'impact sur les cours de la « mauvaise nouvelle» que constitue à priori la publication d'un rapport d'audit avec réserves, ils se sont intéressés aux conséquences boursières d'une information a priori favorable à l'entreprise, à savoir la publication d'un rapport d'audit sans réserves après un ou plusieurs exercices d'opinions réservées. Les auteurs ont ainsi recensé tous les retraits de réserves annoncés entre 1978 et 1987.

\subsection{Construction du modèle}

Comme le souligne DeAngelo (1981), la qualité de l'audit externe repose sur deux concepts fondamentaux : la compétence (qui traduit la capacité de l'auditeur à déceler les anomalies) et l'indépendance (qui est le pouvoir qu'à ce dernier de révéler les anomalies constatées).

Si mesurer la qualité réelle des travaux semble difficile, des études (DeAngelo, 1981 ; Citron et Taffler, 1992 ; Prat dit Hauret, 2003) ont montré qu'elle peut s'appréhender à travers la notion de qualité perçue, basée sur l'apparence. Dans ce cas, on parlera de compétence et/ou d'indépendance perçue(s) de l'auditeur. Il est néanmoins clair que certains facteurs identifiés pour expliquer les différentes approches dans des pays développés ne peuvent tous être pertinents dans un environnement en développement, surtout en raison de la vétusté du système et des institutions.

\subsubsection{Compétence perçue de l'auditeur}

Une revue de la littérature montre que la compétence perçue est appréhendée à l'aide de plusieurs variables, regroupées en deux dimensions : une première prenant en compte les variables liées à l'auditeur et une seconde intégrant les variables liées à l'audité.

Pour les partisans de la première dimension, dont Flint (1988), les auditeurs doivent posséder des connaissances, une formation, une qualification et une expérience suffisante pour mener à bien un audit financier. Lee (1993) s'inscrit à la suite de son prédécesseur et souligne qu'un auditeur est compétent s'il est «suffisamment qualifié et expérimenté pour vérifier la qualité des états financiers et ainsi l'attester auprès des actionnaires et des autres utilisateurs intéressés ».

Il semble que la nature des ressources mobilisées par un auditeur dépende de son grade. Des études (Benner, 1984) ont démontré que les praticiens gagnent en compétences durant les premières années de pratique, périodes pendant lesquelles ils développent l'aptitude au 
raisonnement professionnel et acquièrent une plus grande expertise dans l'application des concepts théoriques aux problèmes de terrain. Dans la même veine, Dreyfus et Dreyfus (1989) proposent un continuum de la compétence qui comprend cinq niveaux, du niveau novice au niveau expert. La compétence s'exprime à travers un vocabulaire cognitiviste, soit la capacité à résoudre des problèmes, l'habileté à apprendre, l'aptitude à la pensée holistique. Elle met en outre l'accent sur la polyvalence et la flexibilité de l'individu.

L'auditeur qui rédige et signe le rapport général d'audit (de même que le rapport « spécial » et le rapport « article $715 »)$ est obligatoirement un commissaire aux comptes. Dans sa mission, ce dernier est assisté d'auditeurs collaborateurs. Avec l'expérience, les collaborateurs d'audit passent d'un grade ${ }^{10}$. Emby et Etherington (1996) ont mesuré l'importance accordée par des auditeurs de tous niveaux à différents critères d'évaluation de la performance de ces derniers à chaque grade. En ce qui concerne les auditeurs de terrain, les critères d'évaluation les plus importants relevés par les auteurs sont les « habiletés techniques » et «l'aptitude à résoudre des problèmes ». Les auditeurs confirmés seraient en plus évalués sur leur «aptitude à la communication » et les seniors sur la « gestion de la mission d'audit » qui fait référence à la répartition et à la supervision des travaux. Quant aux associés, ils seraient également évalués sur « le service au client » et « le développement du cabinet », aspects davantage liés au « business of auditing » qu'au « craft of auditing ».

Les développements précédents soulignent l'existence d'une relation entre le grade et la phase du processus de certification, dans la mesure où tous les auditeurs, quel que soit leur grade, ne peuvent pas réaliser toutes les étapes de la démarche d'audit. Le grade dépend directement des niveaux de formation et d'expérience, ce dernier étant mesuré par le nombre d'années d'exercice en audit. Le niveau de formation fait certes référence à la formation initiale et aux diplômes obtenus (le fait de posséder ou non le diplôme d'Expert-comptable), mais aussi à la formation professionnelle.

La compétence de l'auditeur comme un agent individuel n'est pas le seul déterminant de la compétence du cabinet d'audit. Selon Fama et Jensen (1983), l'organisation du cabinet caractérisé par sa structure hiérarchique, la forme de la surveillance, le contrôle des auditeurs et des équipes d'auditeurs, représente une garantie sur la compétence et la qualité de l'audit. Cette organisation doit être spécifiée en prenant en considération plusieurs paramètres, notamment le fait de travailler ou non en équipe, l'organisation de la coopération entre

\footnotetext{
${ }^{10}$ Dans les grands cabinets : on distingue souvent les grades suivants : débutant, confirmé, senior, manager, senior manager, associé.
} 
compétences individuelles, l'existence ou non d'une revue par les pairs, (par exemple affectation d'un professionnel chevronné à chaque débutant ou coaching...).

Les partisans de la seconde composante, dont Abdolmohammadi et Shanteau (1992), soutiennent une idée avec un fondement basé sur l'expertise, c'est-à-dire, la qualification et l'expérience des auditeurs qui leur permet de réaliser un travail technique sans effort, de manière fluide et intuitive en évitant au maximum les erreurs. Pour les adeptes de cette dimension, deux autres variables semblent jouer un rôle déterminant dans l'organisation du cabinet d'audit et influencer indirectement la nature des ressources mobilisées. Il s'agit du mode d'exercice de la profession et de la taille du cabinet en question. En effet, la nature des ressources mobilisées par un auditeur dépend du type d'entreprise contrôlé (taille, ouverture internationale, structure de propriété, secteur d'activités, connaissance de l'activité...); sachant que les grandes entreprises internationales sont le plus souvent contrôlées par des grands cabinets internationaux et que les commissaires aux comptes exerçant à titre individuel contrôlent le plus souvent les comptes de moyennes entreprises.

En somme, la compétence n'est donc pas à rechercher uniquement chez l'auditeur en action. Elle est le fruit d'une construction conjointe entre l'auditeur, le cabinet d'audit et l'entreprise auditée et traduit ainsi une notion complexe. Tout d'abord, c'est un construit social élaboré et évalué par une pluralité d'acteurs. Ensuite, à partir de ses éléments constitutifs, on distingue en audit la compétence individuelle de la compétence collective.

Toutefois, la qualité de l'audit ne saurait se limiter à la capacité de l'auditeur à mettre en évidence des anomalies. Elle doit également s'étendre à sa capacité à révéler ces anomalies significatives relevées, autrement dit son indépendance.

\subsubsection{L'indépendance perçue de l'auditeur}

Il existe différentes formes d'indépendance et la distinction la plus classique propose d'analyser les notions d'indépendance de fait et d'indépendance d'apparence (Richard, 2003). L'indépendance se réfère alors au processus mental de l'auditeur et à son attitude d'impartialité et d'objectivité. Traditionnellement, elle est perçue comme un état d'esprit. A la suite de Lee (1993), Richard (2003) estime qu'il est délicat de donner un sens opérationnel à ce qui est essentiellement état d'esprit, dans une situation dans laquelle l'auditeur est intellectuellement distant du bénéficiaire de son indépendance.

Même s'il est donc mentalement indépendant, l'auditeur doit donner les signes visibles, explicites et accessibles au public qu'il en est ainsi (Richard, 2003). Toutefois, La relation 
entre les dirigeants et les actionnaires peut conduire à des pressions contradictoires sur l'indépendance de l'auditeur. Ceci amène Flint (1988) à conclure que l'analyse de l'indépendance de l'auditeur dépend de son niveau de probité face aux pressions imposées par ses clients ou relatives à son activité.

Une revue de la littérature montre que les variables de l'indépendance perçue sont regroupées en trois catégories : celles liées respectivement à l'auditeur, à l'audité et à la gouvernance.

\section{Les variables liées à l'auditeur}

Des études (Abdolmohammadi et Wright, 1987) ont montré que lors d'une situation complexe à résoudre pour un auditeur, le niveau d'expérience acquise a un effet significativement positif sur la qualité de la réponse apportée à un problème posé. La compétence étant la capacité reconnue en une matière et qui donne le droit d'en juger, il est nécessaire que l'auditeur dispose d'un ensemble de critères lui permettant de réaliser de manière satisfaisante et efficace toutes les diligences d'audit : être à la fois capable, doté de solides connaissances, suffisamment expérimenté, etc. Prat dit Hauret (2003) montre quant à lui qu'un auditeur peut difficilement être indépendant s'il n'est pas compétent, la compétence de l'auditeur étant une condition nécessaire à son indépendance. La décision de l'auditeur d'être dépendant ou indépendant ne peut être prise que si sa compétence lui permet d'accomplir de manière totalement satisfaisante l'ensemble de ses travaux d'audit. Plus le niveau d'expérience de l'auditeur est élevé, plus il est capable d'apprécier la complexité d'une situation. Il a de ce fait davantage de chances d'être perçu comme plus indépendant qu'un auditeur dont le niveau d'expérience serait plus faible. DeAngelo (1981) estime pour sa part que les cabinets de grande taille disposant d'un portefeuille clients important et diversifié seront moins vulnérables aux pressions d'un client particulier. Dans la continuité de leur prédécesseur, Richard (2003) et Blokdijk et al. (2006) indiquent que la taille du cabinet est un critère d'évaluation de l'indépendance des auditeurs qui offre l'avantage d'être facilement observable par les utilisateurs de l'information financière. Ce critère est logiquement lié à la réputation du cabinet. Au sens de Watts et Zimmerman (1986), la réputation est la raison la plus importante dont dispose l'auditeur pour résister aux pressions de l'équipe dirigeante. Ainsi, le retentissement d'une défaillance pour les cabinets de grande taille entraînant des répercussions négatives sur leur notoriété (réputation), ces derniers sont supposés moins vulnérables aux pressions des clients. Par ailleurs, les cabinets de grande taille sont censés être financièrement plus indépendants que ceux de petite taille. Cette notoriété se base sur des critères quantitatifs objectifs tels que le nombre d'employés, le nombre de clients audités, le 
volume des honoraires facturés en audit ou le nombre d'associés détenant le capital, le pouvoir et les responsabilités. Les grands cabinets seraient alors perçus par les utilisateurs de l'information financière comme plus indépendants que les plus petits car leurs condamnations en dommages et intérêts seraient plus lourdes en cas d'erreur (Prat dit Hauret, 2003). Ils peuvent être également plus facilement la cible de poursuites judiciaires étant donné que leur niveau de couverture en matière d'assurance responsabilité civile professionnelle est plus élevé. Toutefois, la disparition du cabinet Arthur Andersen peut être considérée comme «l'exception qui infirme la règle ».

Outre les éléments sus-cités, Rest (1986) souligne l'importance de la «sensibilité éthique ${ }^{11}$. Selon lui, lors de l'émission d'une opinion de certification, l'auditeur peut être confronté à un véritable dilemme éthique en cas de désaccord avec les dirigeants de l'entreprise : il doit soit renoncer à l'expression de son avis en adoptant un comportement opportuniste pour conserver son mandat, soit exprimer son avis au service de la communauté financière, ce qui peut entraîner la perte de son mandat et des honoraires y afférents. Plus la sensibilité éthique de l'auditeur est perçue comme forte, plus les utilisateurs de l'information comptable et financière le perçoivent comme un acteur indépendant et capable de résister aux pressions dans le cadre de sa mission d'opinion sur les comptes annuels (Prat dit Hauret, 2003). D'autres travaux (Prat dit Hauret, 2000 ; Shafer et al. 2001 ; Hottegindre et Lesage, 2009) indiquent que le niveau d'indépendance est fortement corrélé au niveau d'éthique puisque l'auditeur est libre d'émettre le jugement qu'il désire.

Dans leur étude de l'incidence de la revue ${ }^{12}$ du dossier sur l'avis de l'associé signataire, Matsumura et Tucker (1995) montrent que la révision du dossier par un second associé pousse l'associé signataire non seulement à se comporter avec une plus grande indépendance au moment de l'émission de son opinion, mais également à effectuer des sondages plus nombreux au moment de l'évaluation du contrôle interne et du contrôle des comptes. Cela peut inciter l'associé signataire à adopter un comportement plus indépendant lorsqu'il émet son opinion. Ainsi, le fait que le lecteur des comptes ait connaissance de l'existence des mécanismes incitatifs de contrôle qualité dans les cabinets d'audit contribuerait à rendre l'auditeur plus crédible. C'est ce qui amène Prat dit Hauret (2003) à affirmer que «la

\footnotetext{
${ }^{11}$ Il s'agit de la capacité d'un individu à interpréter une situation donnée et à se rendre compte qu'un problème moral existe.

${ }^{12} \mathrm{La}$ revue par un co-associé (contrôle qualité) est une mesure qui consiste à faire revoir un dossier d'audit par un associé autre que celui chargé dudit dossier.
} 
surveillance mutuelle améliore la qualité du service rendu au client et la compétence de chaque associé du cabinet. »

\section{Les variables liées à l'audité}

Dans le but d'obtenir des honoraires élevés lorsque l'entreprise est en bonne santé, l'auditeur va produire un audit de qualité de par un travail soutenu (Bell et al., 2001). Les conclusions de Palmrose (1987) et de Prat dit Hauret (2003) vont d'ailleurs dans ce sens. Pour ces derniers, la mauvaise situation financière d'un client audité peut constituer un risque d'audit. En effet, les auditeurs en relation avec des clients qui sont en mauvaise posture financière risquent la mise en cause de leur responsabilité civile et pénale. Ce risque est ici beaucoup plus élevé que dans le cas où leurs clients sont en bonne santé financière. Les utilisateurs de l'information financière perçoivent que les dirigeants ont beaucoup plus de possibilités d'obtenir satisfaction en cas de conflit avec l'auditeur si l'entreprise est en bonne santé financière. Un cabinet d'audit qui réalise qu'il y a peu de risques de mise en cause de sa responsabilité, en raison de l'excellente situation financière de son client, tend à être moins motivé pour résister aux pressions des dirigeants de la société auditée. Le risque perçu, par les cabinets, de mise en cause de leur responsabilité, est inversement proportionnel à la santé financière du client. Ceci amène d'aucuns à prétendre que les sociétés en mauvaise santé financière sont tentées de manipuler les comptes et de produire ainsi des états financiers comportant beaucoup plus d'erreurs que ceux des sociétés en bonne santé financière.

Partant de l'idée que le marché de l'audit est très concentré, Pigé (2003) a montré que la pression de la concurrence peut inciter les auditeurs à compromettre leur indépendance. Prat dit Hauret (2003) est d'ailleurs de cet avis. Pour lui, ces cabinets développent un système tacite de récompenses qui valorise les aspects commerciaux de la profession au détriment des composantes essentielles. Cette thèse amène à conclure à une corrélation positive entre l'indépendance et l'environnement compétitif.

\section{Les variables liées à la gouvernance}

Si le Co-commissariat aux comptes renforce l'indépendance, la mise en cause de la responsabilité des auditeurs et, éventuellement, les sanctions judiciaires prononcées à leur encontre permettent également de conforter le niveau d'indépendance perçue (Prat dit Hauret, 2003). 
Dallocchio $(2002)^{13}$ pense plutôt à un effet de rotation des auditeurs. Son étude sur les effets de la règle de rotation des auditeurs tous les 9 ans ${ }^{14}$ et sur l'indépendance des auditeurs permet de constater que le sentiment général autour de ladite règle est plutôt positif, même si l'on ne peut démontrer une amélioration de la qualité des comptes. 69\% des personnes interrogées ont répondu qu'elles étaient d'accord avec cette contrainte imposée aux auditeurs. La principale raison fréquemment invoquée est que la règle d'alternance évite la tendance générale des auditeurs à tomber dans une certaine routine dans le cas d'une relation ancienne avec un client. Même si la section 203 de la SOX prévoit la rotation des auditeurs tous les 5 ans, Prat dit Hauret (2003) estime qu'il peut arriver que les aspects négatifs liés à la rotation des auditeurs sur un même dossier soient plus importants que les aspects positifs associés à la diminution d'une relation de complicité trop forte entre l'auditeur et son client. Cet argumentaire trouve son fondement dans les travaux de Siegel (1999). En fait, la rotation des associés sur un dossier ne saurait être une solution à la problématique de l'indépendance perçue de l'auditeur. Ces divergences d'opinions conduisent à affirmer que la rotation a un impact positif sur l'indépendance perçue alors que, paradoxalement, on observe le contraire pour ce qui est de l'indépendance réelle.

Pour Deis et Giroux (1992), au fur et à mesure qu'augmente la durée du mandat de l'auditeur, la qualité de son opinion et le niveau d'indépendance perçue baissent. A partir d'une certaine durée, l'auditeur n'est plus indépendant. Une durée de mandat longue entraînerait une complaisance croissante, ce qui occasionnerait pour l'auditeur la mise en œuvre de techniques de révision de moins en moins innovantes (Prat dit Hauret, 2003).

Parallèlement aux variables relevées, d'autres études montrent que la publication du montant des honoraires influence positivement l'indépendance perçue. En effet, celui-ci représente la rétribution versée à l'auditeur par son client en contrepartie de sa prestation d'audit. Le fait qu'un lecteur des comptes ait connaissance d'un montant d'honoraires très élevé peut rendre l'auditeur financièrement dépendant de son client. Pour éviter une perte d'honoraires, l'auditeur peut céder à certaines pressions exercées par le client, lequel le menace par exemple de changer de cabinet et s'abstenir ainsi de révéler certaines défaillances comptables. Paradoxalement, un montant d'honoraires très faible augmente le risque de non détection du manque d'intérêt de l'auditeur. Ceci permet d'avancer que plus le montant des honoraires d'audit est élevé, plus l'indépendance est remise en cause.

\footnotetext{
${ }^{13}$ D'après la Lettre Vernimmen.net $n^{\circ} 14$ de novembre 2002. Son étude a porté sur un échantillon de 502 sociétés cotées sur la bourse italienne entre 1992 et 2001.

${ }^{14}$ En Italie, la durée du mandat est de trois ans renouvelables deux fois pour une même firme.
} 
Toutefois, certains auteurs estiment estime que les prestations de conseil peuvent améliorer l'indépendance. Leur argumentaire est basé sur l'idée qu'elles participent à améliorer la connaissance de l'auditeur sur le client et, par conséquent, sa dépendance technique. Cela ne se justifierait que dans le seul cadre de l'indépendance réelle car la connaissance, chez les lecteurs des comptes, de cet état de fait nuirait à sa crédibilité. Le risque de remise en cause de l'opinion de l'auditeur pour des raisons commerciales diminuerait lorsque les équipes chargées de réaliser les missions de conseil sont différentes et séparées des équipes d'audit.

\section{Approche méthodologique de l'étude}

La recherche est menée à partir d'une démarche positiviste. Le terrain d'investigation est le Cameroun. Les utilisateurs d'états certifiés retenus pour l'enquête sont constitués des analystes financiers et de crédit de la Bourse de Douala et des banques de Douala et Yaoundé. On retrouve également dans l'échantillon des contrôleurs internes de gestion (auditeurs internes et contrôleurs de gestion) des grandes sociétés soumises aux obligations légales de certification de leurs comptes, ainsi que des membres d'assemblées générale et/ou conseils d'administration de sociétés et des inspecteurs des impôts, soit six (6) groupes d'utilisateurs que nous avons contactés à raison de 16 utilisateurs par groupe.

Le choix des villes retenues est lié au fait qu'on retrouve la plupart des structures employant des auditeurs internes et des contrôleurs de gestion. En outre, ces villes concentrent l'essentiel des entreprises (environ $85 \%)^{15}$. Après un pré-test du questionnaire auprès de 5 chercheurs et 5 professionnels, une version finale a été établie et envoyée aux utilisateurs réels et potentiels des états certifiés. La version transmise aux inspecteurs des impôts a été légèrement modifiée : à la mention «états financiers certifiés », nous avons préféré « DSF ${ }^{16}$, concept mieux compris par les agents du fisc. Sur les 177 questionnaires administrés, 123 ont été récupérés et 14 d'entre eux se sont avérés invalides puisque renseignés par des personnes ne faisant pas partie de notre cible. Au total, 109 réponses ont été exploitées. Le tableau 1 retrace leurs caractéristiques.

\footnotetext{
${ }^{15}$ Informations fournies par l'Institut national de la statistique (INS).

${ }^{16}$ Déclaration statistique et fiscale.
} 
Tableau 1 : Caractéristiques de l'échantillon

\begin{tabular}{|l|l||l|l}
\hline Effectif & $\begin{array}{l}\text { Fréquence } \\
\%\end{array}$ & $\begin{array}{l}\text { Forme } \\
\text { juridique }\end{array}$ & $\begin{array}{l}\text { Fréquence } \\
\%\end{array}$ \\
\hline Auditeur & 14,5 & SARL & 12,7 \\
\hline $\begin{array}{l}\text { Contrôleur } \\
\text { de gestion }\end{array}$ & 14,5 & SA & 58,2 \\
\cline { 2 - 4 } $\begin{array}{l}\text { Inspecteur } \\
\text { des impôts }\end{array}$ & 16,4 & EPA & 14,5 \\
\hline $\begin{array}{l}\text { Employés de } \\
\text { banque }\end{array}$ & 20 & $\begin{array}{l}\text { Fonction } \\
\text { publique }\end{array}$ & 14,5 \\
\hline Actionnaires & 34,5 & \multicolumn{2}{|l}{} \\
\hline
\end{tabular}

\begin{tabular}{|l|l|}
\hline $\begin{array}{l}\text { Secteur } \\
\text { d'activités }\end{array}$ & $\begin{array}{l}\text { Fréquence } \\
\text { \% }\end{array}$ \\
\hline $\begin{array}{l}\text { Etats } \\
\text { financiers }\end{array}$ & 43,6 \\
\hline Impôts & 14,5 \\
\hline Hôtellerie & 14,5 \\
\hline Energie & 12,7 \\
\hline Imprimerie & 7,3 \\
\hline Transport & 7,3 \\
\hline
\end{tabular}

\section{Résultats et implications}

La présentation des résultats s'est faite en deux étapes. Dans la première, nous avons regroupé l'ensemble des indicateurs de mesure des variables explicatives en facteurs via l'analyse en composantes principales. Via un modèle logit, la seconde étape teste les relations entre les variables manifestes ${ }^{17}$ et la variable à expliquer, ici la perception (positive ou négative) de la qualité des travaux d'audit.

\subsection{Extraction des composantes principales}

Après avoir démontré ${ }^{18}$ que la multi-colinéarité ne pose aucun problème, nous avons réalisé des analyses en composantes principales (ACP) sur chacune des composantes, comme l'indiquent les tableaux 2 et 3.

\subsubsection{Une compétence de l'auditeur définie suivant quatre axes}

Une synthèse des résultats des ACP sur la compétence est consignée dans le tableau ci-après.

Tableau 2: Synthèse de l'ACP de la variable compétence de l'auditeur

\begin{tabular}{|l|l|l|l|l|}
\hline & Fac 1_1 & Fac 2_1 & Fac 3_1 & Fac 4_1 \\
\hline Diplôme du directeur de mission & & 0,963 & & \\
\hline Diplôme des auditeurs seniors & & & 0,886 & \\
\hline Renommée des diplômes du personnel technique & & 0,901 & & \\
\hline Ancienneté du directeur de mission & & & & 0,841 \\
\hline Ancienneté des membres de l'équipe & & & 0,630 & \\
\hline Connaissance de l'activité de l'entreprise & 0,789 & & & \\
\hline Visites fréquentes du directeur de mission sur le site & 0,682 & & & \\
\hline Présence permanente du directeur de mission sur le site & & 0,756 & & \\
\hline
\end{tabular}

17. Il s'agit des principaux facteurs précédemment obtenus.

${ }^{18}$ A partir des tests de corrélation de Pearson. 


\begin{tabular}{|l|l|l|l|l|} 
Respect mutuel au sein de l'équipe d'audit & 0,796 & & & \\
\hline Bonne communication horizontale entre les auditeurs & 0,698 & & & 0,838 \\
\hline Conclusion rédigée en concertation avec la direction & & & & \\
\hline Visites fréquentes du chef de mission & & \multicolumn{4}{|c|}{0,507} & $\mathbf{0 , 7 6 5 3}$ \\
\hline Alpha de Cronbach & \multicolumn{4}{|c|}{$\mathbf{0 , 5 8 5}$} \\
\hline Indice Kaiser-Meyer-Oklin & \multicolumn{4}{|c|}{} \\
\hline Valeurs propres & $\mathbf{4 , 0 8 5}$ & $\mathbf{2 , 3 6 2}$ & $\mathbf{1 , 9 8 9}$ & $\mathbf{1 , 0 1 1}$ \\
\hline$\%$ variance & 34,04 & 19,687 & 16,574 & 8,422 \\
\hline$\%$ variances cumulées & 34,04 & 53,727 & 70,301 & $\mathbf{7 8 , 7 2 2}$ \\
\hline
\end{tabular}

Les valeurs de l'indice KMO et de l'Alpha de Cronbach témoignent d'une bonne fiabilité de l'analyse. Ainsi, l'axe 1 est marqué par le regard beaucoup plus poussé des individus en ce qui concerne la connaissance de l'activité de l'entreprise $(0,789)$, la présence permanente du directeur de mission sur le site $(0,756)$, le respect mutuel au sein de l'équipe d'audit $(0,796)$ et une bonne communication horizontale entre les auditeurs $(0,698)$, ce qui a permis de renommer cet axe gestion attendue de la mission. Suivant le même principe, les autres axes ont ainsi été renommés comme suit : contribution attendue du directeur de mission, encadrement de l'équipe d'audit et coopération avec la direction.

\subsubsection{Une indépendance de l'auditeur expliquée par cinq axes}

Comme dans le cas de compétence de l'auditeur, une analyse en composantes principales est réalisée avec les items de mesures de l'indépendance de l'auditeur. Il en découle cinq principaux axes comme l'indique le tableau 9 ci-après. Chaque item est parfaitement corrélé avec un seul axe. Les loadings sont compris entre 0,621 et 0,978. La fiabilité de cette ACP est démontrée, d'une part, par le test de sphéricité de Bartlett qui affiche un indice KMO de 0,689 et, d'autre part, par la forte valeur du coefficient d'alpha de Cronbach $(0,8267)$. Ceci a permis de renommer ainsi les axes factoriels obtenus : dépendance à l'entreprise (axe 1), situations conflictuelles (axe 2), révélation des anomalies (axe 3), de la réputation de l'auditeur (axe 4) et de la responsabilité sociale des parties prenantes (axe 5).

Tableau 3: Synthèse de l'ACP relatif à l'indépendance de l'auditeur

\begin{tabular}{|l|l|l|l|l|l|}
\hline & Fac 1_2 & Fac 2_2 & Fac 3_2 & Fac 4_2 & Fac 5_2 \\
\hline Membre des Big Four & & & & 0,821 & \\
\hline Implantation internationale du cabinet & & & & 0,917 & \\
\hline Nombre d'associés au sein du cabinet & & & & & 0,731 \\
\hline Résultat déficitaire dans l'entreprise auditée & & 0,978 & & & \\
\hline $\begin{array}{l}\text { Situation financière considérée comme un élément } \\
\text { complémentaire }\end{array}$ & & 0,946 & & & \\
\hline Application des sanctions disciplinaires prévues & & & 0,805 & & \\
\hline Limitation du nombre de mandats consécutifs & & & 0,675 & & \\
\hline
\end{tabular}




\begin{tabular}{|l|l|l|l|l|l|} 
Rotation des équipes d'audit & & & 0,745 & & \\
\hline $\begin{array}{l}\text { Mise en œuvre de missions de conseil et de CAC pour la } \\
\text { même entité }\end{array}$ & 0,855 & & & & \\
\hline Dépendance financière vis-à-vis de la même entreprise & 0,707 & & & & \\
\hline Contrôle administratif externe de la profession d'audit & & 0,621 & & & \\
\hline $\begin{array}{l}\text { Rattachement au ministère de la justice de l'organe du } \\
\text { contrôle externe }\end{array}$ & & & & & 0,879 \\
\hline Absence de normes d'audit propres & & 0,633 & & & \\
\hline Alpha de Cronbach & \multicolumn{5}{|c|}{ KM = 0,8267 } \\
\hline Indice Kaiser-Meyer-Oklin & \multicolumn{5}{|c|}{} \\
\hline Valeurs propres & $\mathbf{3 , 6 6 4}$ & $\mathbf{3 , 1 2 9}$ & $\mathbf{1 , 5 8 8}$ & $\mathbf{1 , 4 2}$ & $\mathbf{1 , 0 6}$ \\
\hline$\%$ variances & 28,182 & 24,072 & 12,216 & 10,925 & 8,156 \\
\hline$\%$ variances cumulées & 28,182 & 52,254 & 64,47 & 75,375 & $\mathbf{8 3 , 5 5 1}$ \\
\hline
\end{tabular}

En définitive, on dénombre neuf composantes principales utilisées comme variables manifestes dans la régression logistique effectuée.

\subsection{Une perception de la qualité de l'audit dictée par plusieurs facteurs}

Suivant les préconisations de Raffournier (1990), nous avons réalisé une régression logistique entre la variable dépendante et les variables indépendantes ou manifestes. Les variables de contrôle sont l'effectif, la forme juridique et le secteur d'activités.

Trois modèles ont été testés, en reprenant pour le premier l'ensemble des variables explicatives. Le second modèle ne retient que les facteurs corrélés aux variables de la compétence de l'auditeur. Le troisième modèle enfin se focalise uniquement sur les facteurs restitués à partir des items de mesure de l'indépendance de l'auditeur. La pertinence de ces régressions a été appréciée via le $\mathrm{R}^{2}$ de Nagelkerke, le rapport de vraisemblance, le taux de classification et le coefficient de Wald, qui sont des indicateurs d'appréciation de la qualité de la régression. 
$\underline{\text { Tableau } 4}$ : Synthèse des résultats de la régression logistique

\begin{tabular}{|c|c|c|c|c|c|c|}
\hline \multirow[b]{2}{*}{ Variables } & \multicolumn{2}{|c|}{$\begin{array}{l}\text { Modèle } 1 \\
\text { (TCT+TRC) }\end{array}$} & \multicolumn{2}{|c|}{$\begin{array}{l}\text { Modèle } 2 \\
\text { (TCT) }\end{array}$} & \multicolumn{2}{|c|}{$\begin{array}{l}\text { Modèle } 3 \\
\text { (TRC + SPE) }\end{array}$} \\
\hline & Coef & Wald & Coef & Wald & Coef & Wald \\
\hline Gestion attendue de la mission & 0,486 & $8,835 * * *$ & 0,470 & $8,579 * * *$ & & \\
\hline Contribution attendue du directeur de mission & 0,452 & $5,578 * *$ & 0,448 & $5,375^{* *}$ & & \\
\hline Encadrement de l'équipe d'audit & 0,080 & 0,647 & 0,065 & 0,573 & & \\
\hline Coopération avec la direction & 0,382 & $4,792 * *$ & 0,373 & $4,437 * *$ & & \\
\hline Dépendance à l'entreprise & 0,268 & 0,269 & & & 0,255 & 0,218 \\
\hline Situations conflictuelles & 0,653 & 0,658 & & & $9,227 * * *$ & $10,131^{* * *}$ \\
\hline Révélation des anomalies & 0,591 & $9,237 * * *$ & & & 0,583 & $10,296^{* * *}$ \\
\hline Réputation de l'auditeur & 0,320 & $4,013 * *$ & & & 0,373 & $4,058^{* *}$ \\
\hline Responsabilité sociale des parties prenantes & 0,258 & $0,227 *$ & & & 0,356 & $3,866^{* *}$ \\
\hline Constante & $-0,449$ & $7,218 * * *$ & $-0,425$ & $7,166^{* * *}$ & $-0,416$ & $7,135 * * *$ \\
\hline Effectif & $-0,631$ & $8,228 * *$ & $-0,612$ & $8,486 * *$ & $-0,591$ & $7,782 * *$ \\
\hline Forme juridique & $-0,389$ & $4,040 *$ & $-0,416$ & $4,624 *$ & $-0,450$ & $5,236^{*}$ \\
\hline Secteur d'activités & 0,328 & $3,038^{*}$ & $-0,287$ & $3,624^{*}$ & 0,269 & $4,182 *$ \\
\hline Constante & 0,359 & $4,679 * *$ & 0,344 & $4,538 * *$ & 0,317 & $4,563 * *$ \\
\hline$\overline{\mathbf{R}^{2} \text { de Nagelkerke }}$ & \multicolumn{2}{|l|}{$\overline{0,405}$} & \multicolumn{2}{|l|}{$\overline{\mathbf{0 , 3 7 8}}$} & \multicolumn{2}{|l|}{$\overline{0,341}$} \\
\hline -2log-vraisemblance & \multicolumn{2}{|l|}{89,256} & \multicolumn{2}{|l|}{112,015} & \multicolumn{2}{|l|}{$\mathbf{1 3 6 , 8 9 7}$} \\
\hline $\mathbf{X}^{2}$ & $\begin{array}{l}X^{2}=75, \\
\left(>X^{2}(8)\right.\end{array}$ & H*** & \multicolumn{2}{|c|}{$\begin{array}{l}X^{2}=49,843^{* * * *} \\
\left(>X^{2}(6)\right)\end{array}$} & \multicolumn{2}{|c|}{$\begin{array}{l}X^{2}=33,597 * * * \\
\left(>X^{2}(3)\right)\end{array}$} \\
\hline Taux de classification & $89 \%$ & & \multicolumn{2}{|l|}{$72,4 \%$} & \multicolumn{2}{|l|}{$67 \%$} \\
\hline
\end{tabular}

Une synthèse des modèles fait apparaître une forte significativité de plusieurs facteurs, comme en témoignent les coefficients de régression et de Wald. En effet, une perception positive de la qualité des travaux d'audit légal est liée à la gestion attendue de la mission, à la contribution escomptée du directeur de mission, à la coopération avec la direction. Les variables associées aux facteurs sont liées au diplôme du directeur de mission et sa renommée, à la concertation avec la direction lors de la rédaction des rapports de mission, au respect entre les membres de l'équipe d'audit. Comme le mentionne à cet effet Flint (1988), les auditeurs doivent posséder des connaissances, une formation, une qualification et une expérience suffisantes pour mener à bien un audit financier. Par ailleurs, les utilisateurs des états certifiés souhaiteraient une présence permanente du directeur de mission sur le site. Il en 
est de même du respect mutuel qui doit exister entre les membres de l'équipe d'audit. Tout ceci renforcerait les liens entre coéquipiers et favoriserait ainsi le bon déroulement de la mission.

Parmi ces facteurs, le diplôme du directeur de mission apparaît comme le vecteur majeur de crédibilité qui décrit le mieux la compétence perçue de l'auditeur. Après avoir effectué des analyses en composantes principales, Lee (1993) et Herrbach (2000) ont obtenu des résultats similaires avec des coefficients légèrement différents.

Il apparaît également que les utilisateurs des états financiers accordent une attention particulière à la situation financière de l'audité, à sa capacité à révéler les anomalies, à la réputation du cabinet, de même qu'à la responsabilité sociale des parties prenantes à l'activité d'audit. Les variables concernées sont : l'appartenance à un cabinet membre Big four, l'implantation internationale du cabinet, le fait que l'entreprise auditée présente un résultat déficitaire ou non, la rotation des équipes d'audit, la limitation du nombre de mandats consécutifs et l'application des sanctions judiciaires.

Ces résultats s'inscrivent dans la continuité de ceux de nombre d'auteurs parmi lesquels : DeAngelo (1981), Knapp (1985), Watts et Zimmerman (1986). Nous rejoignons également les conclusions des études de Palmrose (1987), Richard (2003), Ghorbel Hakim (2009).

\section{Conclusion}

$\mathrm{Au}$ terme de cet article dont l'objet était d'identifier les facteurs explicatifs de la qualité perçue des travaux d'audit par les utilisateurs des états financiers certifiés, plusieurs constats méritent d'être faits. La gestion attendue de la mission, la contribution escomptée du directeur de mission, l'encadrement de l'équipe d'audit et la coopération avec la direction de l'entité auditée semblent constituer autant d'éléments caractérisant au mieux la compétence de l'auditeur, susceptibles d'influencer progressivement ou radicalement l'appréciation qualitative des travaux d'audit par les utilisateurs des états certifiés au Cameroun. En outre, les facteurs liés à l'indépendance perçue de l'auditeur tels que l'indépendance financière, la révélation des anomalies, la réputation de l'auditeur et le renforcement de la crédibilité des parties prenantes de l'activité d'audit constitueraient des vecteurs majeurs de la qualité perçue des travaux d'audit dans un pays en développement comme le Cameroun. 
Même si la perception de la qualité de l'audit légal ne se limite pas uniquement aux variables mobilisées dans l'étude, il est indéniable que cette étude a le mérite d'aborder la question dans un contexte réputé difficile et hostile à la recherche. Aussi, l'accès aux entreprises est rendu difficile par leurs responsables, surtout lorsqu'ils pensent qu'une recherche peut s'avérer contraire à leurs intérêts et à ceux de leurs compagnies. Ils craignent le plus souvent que les données et résultats issus de cette recherche soient utilisés hors de leur contexte. Une telle étude revêt également une importance à plusieurs niveaux : elle constitue un outil d'aide aux préoccupations des contrôleurs internes de gestion (auditeurs internes et contrôleurs de gestion) des grandes sociétés soumises aux obligations légales de certification de leurs comptes, ainsi que des membres d'assemblées générale et/ou conseils d'administration de sociétés et des inspecteurs des impôts, lesquels doivent régulièrement s'interroger sur la qualité de leurs missions.

Si cette étude a pu améliorer la compréhension des raisons guidant la perception des utilisateurs des états certifiés quant à la qualité des travaux dans un pays en développement comme le Cameroun, elle ne constitue bien évidemment qu'un propos d'étape. Certes satisfaisants, les résultats obtenus seraient sans doute meilleurs sur un échantillon plus grand, notamment avec la prise en compte des entreprises du secteur boursier et d'autres catégories reparties sur l'étendue du territoire national. Par ailleurs, en admettant qu'une perception qui relève beaucoup plus de l'apparence peut être très loin de la réalité, on peut se demander quels sont les déterminants de la qualité réelle des travaux d'audit au Cameroun. Ainsi, une étude s'intéressant à la mesure de l'impact de la structure concurrentielle sur l'éthique de l'auditeur serait sans doute plus fructueuse et révélatrice.

\section{Bibliographie}

Abdolmohammadi, M.J., Wright, A. (1987). An examination of the effects of experience and task complexity on audit judgments. The Accounting Review 62(1) : 1-13.

Abdolmohammadi, M.J., Shanteau, J. (1992). Personal attributes of expert auditors. Organisational Behavior and Human Decision Processes 53(2) : 158-173.

Antle, R. (1982). Auditor independence. Journal of Accounting Research 22(1) : 1-20.

Baskin, E. (1972). The communicative effectiveness of consistency exceptions. The Accounting Review 47(1) : 38-51. 
Blokdijk, H., Drieenhuizen, F., Simunic, D.A, Stein, M.T. (2006). An analysis of cross-sectional Differences in Big and Non-Big Public Accounting Firms' audit Programs. Auditing: A Journal of Pratice \& Theory 25(1) : 27-48.

Benner, P. (1984). From Novice to Expert Excellence and Power in Clinical Nursing Practice. London: Adison-Wesley.

Ben Saad, E., Lesage, C. (2007). Des facteurs d'indépendance à un système d'indépendance : proposition d'une nouvelle grille d'analyse de l'indépendance de l'auditeur. $28 \mathrm{e}$ congrès annuel de l'Association francophone de comptabilité, Poitiers.

Audousset-Coulier, S. (2008). La publication des honoraires d'audit par les sociétés cotées françaises : deux études de déterminants. Les déterminants du caractère volontaire de la publication des honoraires d'audit et les déterminants du montant des honoraires d'audit publiés. Doctorat en Sciences de gestion, Paris : HEC Paris

Citron, D.B, Taffler, R.J. (1992). The audit report under going concern uncertainties: an empirical analysis. Accounting and Business Research 22(88) : 337- 345.

Commission européenne (2003). Renforcer le contrôle légal des comptes dans l'Union européenne. Communication de la Commission au Conseil et au Parlement européen, 21 mai 2003.

Compagnie nationale des commissaires aux comptes. Normes-commentaires, déontologie. Paris : CNCC, 2000.

Coriat, B., Weinstein, O. (1995). Les nouvelles théories de l'entreprise, Paris, librairie générale française, 218 pages.

DeAngelo, L.E, (1981). Auditor independence, 'low baling', and disclosure regulation. Journal of Accounting and Economics 3(2): 113-127.

Deis, D.R., Giroux, G.A. (1992). Determinants of audit quality in the public sector. The Accounting Review 67(3) : 462-479.

Dreyfus, H.L, Dreyfus, S.E. (1989). Mind over machine: the power of human intuition and expertise in the era of the computer. Oxford : Basil Blackwell.

Dumontier, P., Raffournier, B. (1989). L'information comptable : Pour qui ? pourquoi ? Revue Française de Gestion, mars.

Elliott, J.A. (1982). "Subject to" audit opinions and abnormal security returns: outcomes and ambiguities. Journal of Accounting Research 20(2) : 617-638.

Emby, C., Etherington, L.D. (1996). Performance evaluation of auditors: Role perceptions of superiors and subordinates. Auditing: A Journal of Pratice \& Theory 15(1) : 99-110.

Fama, E.F., Jensen, M.C. (1983). Separation of ownership and control. Journal of Law and Economics 26(2): 301-325.

Fields, Wilkins, M. (1991). The information content of withdrawn audit qualifications: new evidence on the value of "subject-to" opinions. Auditing: A Journal of practice and Theory 10:2, 62-69. 
Firth, M. (1978). Qualified audit reports: their impact on investment decisions. The Accounting Review $53(3): 642-650$.

Flint, D. (1988). Philosophy and principles of auditing. Basingstoke: Macmillan Education.

France. Loi n ${ }^{\circ}$ 2003-706 du 1er août 2003 de sécurité financière. Journal Officiel de la République Française $177: 13220$.

Gillet, P. (1998). Pour une écologie du concept de compétence. Education Permanente 135(1998-2) : 23-32.

Hazgui, M., Manita, R., Pochet, C. (2010). Les défaillances affectant la qualité de l'audit: une étude qualitative sur le marché français. Colloque de l'Association Francophone de la Comptabilité. Nice, France.

Herrbach, O. (2000). Le comportement au travail des collaborateurs de cabinets d'audit financier. Doctorat en sciences de gestion, Toulouse : Université des Sciences sociales Toulouse I.

Hottegindre, G., Lesage, C. (2009). Un mauvais auditeur: manque d'indépendance et/ou de compétence ? Etude exploratoire des motifs de condamnation des commissaires aux comptes sur le marché de l'audit en France. Comptabilité Contrôle Audit 15(2) : 87-113.

Jensen, M.C., Meckling, W.H. (1976). Theory of the firm: managerial behavior, agency costs and ownership structure. Journal of Financial Economics 3(4) : 305-360.

Knapp, M.C. (1985). Audit conflict: an empirical study on the perceived ability of auditors to resist management pressure. The Accounting Review 60(2) : 202-211.

Lee, T.A. (1993). Corporate audit theory. New York : Chapman \& Hall.

Matsumura, E.M., Tucker, R.R. (1995). Second partner review: an analytical model. Journal of Accounting, Auditing and Finance 10(1) : 173-200.

Ng, D.S., Stoeckenius, J. (1979). Auditing: incentives and truthful reporting. Journal of Accounting Research $17: 1-24$

Palmrose, Z.V. (1987). Litigation and independent auditors: the role of business failures and management fraud. Auditing: A Journal of Practice and Theory 6(2) : 90-103.

Pigé B. (2003). Les enjeux du marché de l'audit. Revue française de gestion 29(147): 87-103.

Prat dit Hauret, C. (2000). L'indépendance du commissaire aux comptes : cadre conceptuel et analyse empirique. Doctorat en sciences de gestion, Bordeaux : Université Montesquieu Bordeaux IV.

Prat dit Hauret, C. (2003). L'indépendance du commissaire aux comptes : une analyse empirique fondée sur trois composantes psychologiques du comportement. Comptabilité Contrôle Audit 9(2) : $31-58$.

Raffournier, B. (1990). La théorie "positive" de la comptabilité: une revue de la littérature. Economie et societies (16) : 137-166

Rest, J.R. (1986). Moral development: advances in research and theory. New York : Praeger.

Richard, C. (2003). L'indépendance de l'auditeur : pairs et manques. Revue française de gestion 147(6) : 119-131. 
Siegel, H. (1999). The LPA: never underestimate the value of a good reputation. The CPA Journal 69(9) : 18

Soltani, B. (1996). Le commissaire aux comptes et le marché financier. Paris : Economica.

Watts, R.L., Zimmerman, J.L. (1986). Positive accounting theory. Englewoods Cliffs : Prentice-Hall. 